\title{
Gravitational Core-Mantle Coupling and the Acceleration of the Earth
}

\author{
by \\ David Parry Rubincam \\ Geodynamics Branch, Code 921 \\ Laboratory for Terrestrial Physics \\ NASA's Goddard Space Flight Center \\ Greenbelt, MD 20771 \\ voice: $301-614-6464$ \\ fax : 301-614-6522 \\ email: rubincam@core2.gsfc.nasa.gov
}

\begin{abstract}
Gravitational core-mantle coupling may be the cause of the observed variable acceleration of the Earth's rotation on the 1000 year timescale. The idea is that density inhomgeneities which randomly come and go in the liquid outer core gravitationally attract density inhomogeneities in the mantle and crust, torquing the mantle and changing its rotation state. The corresponding torque by the mantle on the core may also explain the westward drift of the magnetic field of $0.2^{\circ}$ per year. Gravitational core-mantle coupling would stochastically affect the rate of change of the Earth's obliquity by just a few per cent. Its contribution to polar wander would only be about $0.5 \%$ the presently observed rate.
\end{abstract}


Tidal friction is slowing down the rotation of the Earth, overwhelming a smaller positive acceleration from postglacial rebound. According to historical records such as ancient and medieval observations of solar eclipses $(1,2)$, the signal from these two secular effects appears to be significantly corrupted by a source (or sources) of an episodic or perhaps periodic nature operating on a 1000 year timescale (Figure 1). This nonsecular source causes accelerations which are the same order-of-magnitude as the tides, $\sim 6 \times 10^{-22} \mathrm{~s}^{-}$ 2. Coupling between the liquid outer core of the Earth and the mantle has long been a suspected reason for changes in the length-of-day $(3,4)$. Research heretofore has been aimed at viscous, electromagnetic, and topographic coupling at the core-mantle boundary (5), mostly on the decadal timescale, and at gravitational coupling not related to the problem outlined below (6).

The present investigation focusses on the gravitational coupling between the density anomalies in the convecting liquid outer core and those in the mantle and crust as a possible cause for the observed nonsecular acceleration on the millenial timescale. (Hereafter I use "mantle" as a shorthand for "mantle and crust.") The basic idea is as follows (Figure 2). There are density inhomogeneities caused by blobs circulating in the outer core like the blobs in a lava lamp $(7,8)$; thus the outer core's gravitational field is not featureless. Moreover, these blobs will form and dissipate somewhat randomly. Thus there will be a time variability to the field. These density inhomogeneities will gravitationally attract the density anomalies in the mantle.

The outer core and mantle may not be in a state of gravitational equilibrium if the blobs form and dissipate faster than either the outer core or mantle can react to achieve a state of zero torque. In other words, the mantle and outer core may always be in a state of disequilibrium due to the stochastic nature of "core weather." The outer core and mantle will try to align with each other to make the torque between them zero; but before this can happen, the gravitational field of the outer core may change, so that the core and mantle never catch up with each other. Hence it may be that the outer core is forever torquing the 
mantle, changing its rotation state and perhaps producing the observed nonsecular acceleration and changing the length-of-day. By action-reaction the outer core will also change its rotation state. A quantitive estimate of this effect follows.

The gravitational potential at a point $(r, \theta, \lambda)$ exterior to the Earth can be expressed in the usual spherical harmonic form

$$
V(r, \theta, \lambda)=\frac{G M_{E}}{r} \sum_{\ell=0}^{\infty}\left(\frac{R_{E}}{r}\right)^{\ell} \sum_{m=0}^{\ell} P_{\ell m}(\cos \theta)\left(C_{\ell m} \cos m \lambda+S_{\ell m} \sin m \lambda\right)
$$

where $r$ is the radial distance from the center of mass, $\theta$ is the colatitude, $\lambda$ is the east longitude, and $G=6.673 \times 10^{-11} \mathrm{~m}^{3} \mathrm{~kg}^{-1} \mathrm{~s}^{-2}$ is the universal constant of gravitation, while $M_{E}$ $=5.974 \times 10^{24} \mathrm{~kg}$ is the mass and $R_{E}=6.378 \times 10^{6} \mathrm{~m}$ is the equatorial radius of the Earth (9). The $P_{\ell m}(\cos \theta)$ are the associated Legendre polynomials, while the $C_{\ell m}$ and $S_{\ell m}$ are unnormalized spherical harmonic coefficients. Equation (1) can be more compactly written

$$
V=\frac{G M_{E}}{r} \sum_{\ell m i}\left(\frac{R_{E}}{r}\right)^{\ell} C_{l m i} Y_{l m i}(\theta, \lambda)
$$

where $Y_{\ell m 1}(\theta, \lambda)=P_{\ell m}(\cos \theta) \cos m \lambda, Y_{\ell m 2}(\theta, \lambda)=P_{\ell m}(\cos \theta) \sin m \lambda$, and

$$
C_{\ell m i}=\frac{\left(2-\delta_{0 m}\right)(\ell-m) !}{(\ell+m) !} \frac{\int \rho(r, \theta, \lambda) r^{\ell} Y_{\ell m i}(\theta, \lambda) d \nu}{M_{E} R_{E}^{\ell}}
$$

with $C_{\ell m 1}=C_{\ell m}$ and $C_{\ell m 2}=S_{\ell m}$. Here $\rho(r, \theta, \lambda)$ is the density and $d v$ is an element of volume; the integral is over the whole Earth. The spherical harmonics are normalized according to the following equation: 


$$
\int\left[Y_{\ell m i}(\theta, \lambda)\right]^{2} d A=\frac{4 \pi(\ell+m) !}{\left(2-\delta_{0 m}\right)(2 \ell+1)(\ell-m) !}
$$

where $d A$ is an element of area on the unit sphere. Likewise, by analogy with (2) the gravitational potential from the core alone can be written

$$
V_{C}=\frac{G M_{C}}{r} \sum_{\ell m i}\left(\frac{R_{C}}{r}\right)^{\ell} K_{\ell m i} Y_{\ell m i}(\theta, \lambda)
$$

where $M_{C}=1.947 \times 10^{24} \mathrm{~kg}$ is the mass and $R_{C}=3.480 \times 10^{6} \mathrm{~m}$ is the radius of the core, with the $K_{\ell m i}$ being the spherical harmonic coefficients of the core.

The density anomalies in the core will attract the density anomalies in the mantle, producing a torque $\overrightarrow{\mathbf{L}}$ on the mantle. An element of torque will be given by

$$
d \overrightarrow{\mathbf{L}}=\rho(r, \theta, \lambda) d v\left(\overrightarrow{\mathbf{r}} \times \vec{\nabla} V_{C}\right)
$$

where $\overrightarrow{\mathbf{r}}$ is a point in the mantle and

$$
\overrightarrow{\mathbf{r}}=r(\sin \theta \cos \lambda \hat{\mathbf{x}}+\sin \theta \sin \lambda \hat{\mathbf{y}}+\cos \theta \hat{\mathbf{z}})
$$

Here $\hat{\mathbf{x}}, \hat{\mathbf{y}}$, and $\hat{\mathbf{z}}$ are unit vectors in a right-handed coordinate system fixed in the mantle, with $\hat{\mathbf{z}}$ lying along the Earth's rotation axis and $\hat{\mathbf{x}}$ lying in the equator at zero longitude. For the first few harmonics the expression $\overrightarrow{\mathbf{r}} \times \vec{\nabla} V_{C}$ can easily be found from the Cartesian formulation of the unnormalized associated Legendre polynomials. Writing $Y_{201}(\theta, \lambda)=$ $\left(2 z^{2}-x^{2}-y^{2}\right) / 2$, etc., where $r^{2}=x^{2}+y^{2}+z^{2}$, results in such expressions as 


$$
\overrightarrow{\mathbf{r}} \times \vec{\nabla}\left(\frac{2 z^{2}-x^{2}-y^{2}}{2 r^{4}}\right)=\left(\frac{3 y z}{r^{4}}\right) \hat{\mathbf{x}}-\left(\frac{3 x z}{r^{4}}\right) \hat{\mathbf{y}}
$$

etc., so that $\overrightarrow{\mathbf{r}} \times \vec{\nabla} V_{c}$ can be written

$$
\begin{aligned}
\overrightarrow{\mathbf{r}} \times \vec{\nabla} V_{C}= & \frac{G M_{C}}{R_{C}} \sum_{l m i}\left(\frac{R_{C}}{r}\right)^{\ell+1} Y_{l m i}(\theta, \lambda) \\
& \cdot\left[L_{l m i}^{x} \hat{\mathbf{x}}+L_{l m i}^{v} \hat{\mathbf{y}}+L_{l m i}^{z} \hat{\mathbf{z}}\right]
\end{aligned}
$$

where the $L_{t m i}^{x}, L_{t m i}^{y}$, and $L_{t m i}^{z}$ are coefficients given in Table 1. Integrating (6) gives the the total torque on the mantle:

$$
\overrightarrow{\mathbf{L}}=\int_{R_{\mathrm{c}}}^{R_{E}} \int_{0}^{\pi} \int_{0}^{2 \pi} \rho(r, \theta, \lambda)\left(\overrightarrow{\mathbf{r}} \times \vec{\nabla} V_{C}\right) r^{2} \sin \theta d \lambda d \theta d r
$$

The density can also be expressed in terms of spherical harmonics:

$$
\rho(r, \theta, \lambda)=\sum_{\ell m i} \rho_{\ell m i}(r) Y_{\ell m i}(\theta, \lambda)
$$

By (7)-(9) the component of the torque along the rotation axis $L_{z}$ can be written

$$
L_{z}=\frac{4 \pi(\ell+m) !}{\left(2-\delta_{0 m}\right)(2 \ell+1)(\ell-m) !} G M_{C} R_{C} \sum_{\ell n i i} L_{\ell m i}^{z} \int_{R_{C}}^{R_{E}} \rho_{\ell m i}(r)\left(\frac{R_{C}}{r}\right)^{\ell-1} d r
$$

with similar expressions for $L_{x}$ and $L_{y}$. 
The nonsecular acceleration will be examined first. The time rate of change in the mantles's rotation rate $\Omega$ will be given by

$$
C_{M} \dot{\Omega}=L_{z}
$$

where $C_{M}$ is the moment of inertia of the mantle and currently $\Omega=7.29 \times 10^{-5} \mathrm{~s}^{-1}$.

What is needed now is an estimate of the integral in (10) to use in (11). I will assume here that $\left|K_{\ell m i}\right|<<\left|C_{\ell m i}\right|$ for all coefficients (except perhaps the $\ell=1$ ) terms, so that the $C_{\ell m i}$ coefficients refer to the mantle alone with a high degree of approximation. Moreover, assuming all the mantle density anomalies lie at the Earth's surface probably tends to minimize the torque. Hence from (3) and (9)

$$
C_{\ell m i}=\frac{4 \pi \int_{0}^{R_{E}} \rho_{\ell m i}(r) r^{\ell+2} d r}{(2 \ell+1) M_{E} R_{E}^{\ell}}
$$

and setting $r=R_{E}$ in the above equation and (10) yield

$$
L_{z} \approx \frac{G M_{C} M_{E}}{R_{E}} \sum_{\ell m i} \frac{(\ell+m) !}{\left(2-\delta_{0 m}\right)(\ell-m) !} L_{t m i}^{z} C_{\ell m i}\left(\frac{R_{C}}{R_{E}}\right)^{\ell}
$$

as a conservative estimate of the torque by the core density anomalies on the mantle anomalies. The largest $C_{\ell m i}$ are the second degree terms. Setting $\ell=2$ in (12), writing $M_{C}$ $\approx M_{E} / 3$, and $5\left(R_{C} / R_{E}\right)^{2} \approx 1.5$ in that equation along with $C_{M} \approx M_{E} R_{E}{ }^{2} / 3$ in (11) give, with the aid of the entries in Table 1, 


$$
\dot{\Omega} \approx 7.2 \frac{G M_{E}}{R_{E}^{3}}\left(K_{222} C_{221}-K_{221} C_{222}\right)
$$

as the approximate nonsecular acceleration of the Earth. The coefficients are known to be $C_{221}=1.57 \times 10^{-6}$ and $C_{222}=-0.9 \times 10^{-6}(10)$; since only the order-of-magnitude is important here, they will be taken to be $10^{-6}$. The coefficients $K_{221}$ and $K_{222}$ are unknown as to sign, but the magnitude of the density anomalies in the core are expected to be on the order of $\Delta \rho / \rho=10^{-8}-10^{-9}$, assuming the anomalies are spread evenly throughout the core (11). Using the smaller value in the equation analogous to (12) gives core coefficients $K_{2 m i}$ on the order of $10^{-10}$. Plugging such values in (14) yield values of $\dot{\Omega} \approx 7 \times 10^{-22} \mathrm{~s}^{-2}$, which is the observed order-of-magnitude for the nonsecular acceleration. If characteristic core flow is $10^{-4} \mathrm{~m} \mathrm{~s}^{-1}(7)$, and the blobs dissipate after traveling across the core (about 3000 $\mathrm{km})$, then the intrinsic anomaly timescale of the core variable field will be $\sim 1000$ years.

Suppose that the core gravitational field is responsible for the variable nontidal acceleration of the Earth's rotation and that this acceleration acts on the observed 1000 year time scale. A typical change in mantle rotation speed will be the acceleration $\times$ time. The acceleration has been positive (2) for about the last 1000 years (see Figure 1). so that the change works out to be (ignoring other torques) about $+0.03^{\circ}$ per year in recent times. By action-reaction the torque will slow down the core, which will respond 10 times more quickly to the torque than the mantle, due to the core's smaller moment of inertia (assuming it behaves like a rigid body), and in a sense opposite to the mantle. Hence one would expect the core to drift westward relative to the mantle at a typical speed of 10 times faster, which works out to be $0.3^{\circ}$ per year. The observed drift of the magnetic field is westward at about $0.2^{\circ}$ per year (12), in good agreement with this mechanism. Moreover, the sign and rate of drift can be expected to vary; this is also observed (12) and occurs on the 1000 year time scale, in rough agreement with the nonsecular change in Earth rotation rate. (The speed at 
which the core's lumpy gravitational field washes over the mantle depends on the strength of the coupling and introduces another timescale apart from the intrinsic anomaly timescale over which the torque varies, but at $0.3^{\circ}$ per year this is also happens to be about 1000 years.)

The core-mantle gravitational torque will also affect the obliquity $\varepsilon$, which is the tilt of the Earth's equator with respect to the ecliptic. Here the biggest contributor to the torque will come from the core field acting on the rotational flattening of the mantle, embodied by the $J_{2}=-C_{201}=1 \times 10^{-3}$ coefficient in the external field, which is about a thousand times bigger than any other terms in the field (10). In this case the rate of change of the mantle's obliquity with time will be given approximately by

$$
\dot{\varepsilon}=\frac{L_{x} \hat{\mathbf{x}}+L_{y} \hat{\mathbf{y}}}{C \Omega} \approx \frac{G M_{E} J_{2}}{R_{E}^{3} \Omega}\left(K_{212} \hat{\mathbf{x}}-K_{211} \hat{\mathbf{y}}\right)
$$

which amounts to about $0.5 \mathrm{~m} \mathrm{yr}^{-1}$. The present rate due to the Earth's precession and orbital perturbations is about $14.7 \mathrm{~m} \mathrm{yr}^{-1}$ (13), so that core-mantle coupling accounts for only about $3 \%$ and does no great violence to the expected total. Being an internal torque, the rate of change of the obliquity of the core will be about ten times faster (about $5 \mathrm{~m} \mathrm{yr}^{-1}$ ) because of its smaller moment of inertia, and in the opposite direction.

The core-mantle gravitational torque also produces polar wander. The magnitude of this wander is governed by the Liouville equations (14):

$$
\begin{aligned}
& \dot{m}_{x}+\sigma_{r} m_{y}=\frac{\sigma_{r}}{\Omega^{2}} \frac{L_{x}}{(C-A)} \\
& \dot{m}_{y}-\sigma_{r} m_{x}=-\frac{\sigma_{r}}{\Omega^{2}} \frac{L_{y}}{(C-A)}
\end{aligned}
$$


$m_{x}$ and $m_{y}$ are the $x$ - and $y$-components of the spin vector divided by $\Omega$, and where

$$
\sigma_{r}=\left(\frac{C-A}{A}\right) \Omega \text {. }
$$

These equations ignore all effects on the pole except the torques. Here $A$ is the Earth's moment of inertia measured about an axis in the equator, and $(C-A) / A=1 / 300$, so that $\sigma_{r}$ is a frequency with a period of about 1 year. Differentiating (15) with respect to time and using (16) in the resulting equation gives

$$
\ddot{m}_{x}+\sigma_{r}^{2} m_{x}=\frac{\dot{L}_{x}}{A \Omega}+\sigma_{r} \frac{L_{y}}{A \Omega}
$$

To make this equation easy to solve, I assume that the torque has the form $L_{x}=L_{x}^{0} e^{-\alpha t}$ with a similar expression for $L_{y}$, so that the torque varies on a characteristic timescale of $\alpha^{-1}$. Since this timescale is assumed to be hundreds of years or more, $\alpha<<\sigma_{r}$. Thus the first term on the right side of (17) can be ignored compared to the second. Assuming a particular solution $m_{x}=m_{x}^{0} e^{-\alpha t}$ gives approximately

$$
m_{x}^{0} \approx \frac{L_{y}^{0}}{\sigma_{r} A \Omega}
$$

with a similar expression for the $y$-component. Differentiation the particular solution and using the expressions analogous to (13) yield a rate of polar wander of 


$$
\dot{m}_{x} \hat{\mathbf{x}}+\dot{m}_{y} \hat{\mathbf{y}} \approx \frac{\alpha}{\sigma_{r}} \frac{G M_{E}}{\Omega R_{E}^{3}} J_{2}\left(K_{212} \hat{\mathbf{x}}-K_{211} \hat{\mathbf{y}}\right)
$$

which is once again dominated by the rotational flattening term $J_{2}$. For $K_{21 i} \approx 10^{-10}$ and $\left(\alpha / \sigma_{r}\right) \approx 10^{-3}$ the rate of polar wander is only about $0.05 \mathrm{~cm} \mathrm{yr}^{-1}$, which is well below the observed $10 \mathrm{~cm} \mathrm{yr}^{-1}$ thought to be induced by postglacial rebound.

Gravitational core-mantle coupling appears to be a candidate for explaining the fluctuations observed on the 1000 year timescale in the Earth's rotation rate. It may also explain the westward drift of the core-relative to the mantle, and would add a stochastic component to the rate of change of the mantle's obliquity at perhaps the $3 \%$ level. However, this mechanism would not be able to explain any substantial north-south drift of the magnetic pole relative to the mantle, since this is given by the change in the core's obliquity relative to the mantle, which would only be about $5 \mathrm{~m} \mathrm{yr}^{-1}$. The contribution to the presently observed polar wander is tiny, only about $0.5 \%$.

The above calculations assume that the viscous drag of the outer core on the mantle is negligible. The actual viscosity is unknown. The characteristic spin-up time for a core rotating at a speed different from the mantle is between 10 years and $10^{5}$ years for a plausible range of viscosities (15). Thus only for the lower estimates of viscosity is its neglect justified.

The calculations also assume that the outer core rotates like a rigid body relative to the mantle, when in fact it is a convecting liquid. Further, the convecting blobs in the outer core will exert pressure and electromagnetic as well as viscous forces at the core-mantle boundary $(5,16)$; these are not including here. The sum total of these effects represents future avenues of research, as do the consequences of this coupling for the long-term obliquity of the Earth $(4,17)$ and the rotation of the solid inner core $(18,19)$. 


\section{References and Notes}

1. F. R. Stephenson and L. V. Morrison, Phil. Trans. R. Soc. Lond. A313, 47 (1984).

2. Figure 7 of F. R. Stephenson and L. V. Morrison, Phil. Trans. R. Soc. Lond. A351, 165 (1995).

3. J.-L. Le Mouel, D. E. Smylie, and T. Herring (eds.), Dynamics of Earth's Deep Interior and Earth's Rotation, Amer. Geophys. Un., Geophys. Mono. 72, 1993; K. Lambeck, The Earth's Variable Rotation, Cambridge Univ., Cambridge, 1980.

4. F. D. Stacey, Geophys. J. R. astr. Soc. 33, 47 (1973).

5. M. Lefftz, R. Sabadini, and H. Legros, Geophys. J. Int. 117, 1 (1994).

6. J. Getino, J., Geophys. J. Int. 120, 693 (1995); S. Xu and A. M. K. Szeto, Geophys. J. Int. 118, 94 (1994); S. Xu, D. Crossley, and A. M. K. Szeto, Phys. Earth. Planet. Int. 117, 95-110, 2000; J. Aurnou and P. Olson, Phys. Earth. Planet. Int. 117, 111-121, 2000.

7. H. K. Moffat and D. E. Loper, Geophys. J. Int. 117, 394 (1994).

8. B. A. Buffet, H. E. Huppert, J. R. Lister, and A. W. Woods, Nature 356, 329 (1992); J. Vanyo, P. Wilde, P. Cardin, and P. Olson, Geophys. J. Int. 121, 136 (1995); J. M. Wahr and D. de Vries, Geophys. J. Int. 99, 511 (1989).

9. W. M. Kaula, Theory of Satellite Geodesy, Blaisdell, Waltham, Mass., 1966; M. Caputo, The Gravity Field of the Earth, Academic Press, New York, 1967. 
10. F. J. Lerch, F. J., S. M. Klosko, R. E. Laubscher, and C. A. Wagner, J. Geophys. Res. 84, 3897, (1979). Lerch et al. use normalized coefficients, so that $C_{221}=\sqrt{5 / 12} \bar{C}_{22}$ and $C_{222}=\sqrt{5 / 12} \bar{S}_{22}$, where the bar denotes Kaula's $4 \pi$ normalization.

11. C. V. Voorhies, J. Geophys. Res. 100, 10,029 (1995); C. V. Voorhies, private communication, 1996.

12. F. D. Stacey, Physics of the Earth, Third Edition, Brookfield Pres, Brisbane, Australia, 1992.

13. D. P. Rubincam, B. F. Chao, and B. G. Bills, Sky \& Telescope 95 [6], 36 (1995).

14. W. Munk and G. J. F. MacDonald, The Rotation of the Earth, Cambridge Univ., Cambridge, 1975.

15. B. G. Bills, J. Geophys. Res. 144, 2653 (1999).

16. M. Fang, B. H. Hager, and T. A. Herring, Geophys. Res. Lett. 23, 1493 (1996).

17. M. G. Rochester, Geophys. J. R. astr. Soc. 46, 109 (1976).

18. X. Song and P. G. Richards, Nature 382, 221 (1996).

19. I thank Bruce G. Bills for valuable discussions. 
Table 1. The coupling coefficients $L_{\ell m i}^{x}, L_{\ell m i}^{v}, L_{\ell m i}^{z}$ of equation (7) for $\ell=2$.

$\begin{array}{ccccc}m & i & L_{2 m i}^{x} & L_{2 m i}^{y}, & L_{2 m i}^{z} \\ & & & & \\ 0 & 1 & +K_{212} & -K_{211} & 0 \\ 1 & 1 & +K_{222} / 2 & +3 K_{201}-K_{221} / 2 & -K_{212} \\ 1 & 2 & -3 K_{201}-K_{221} / 2 & -K_{222} / 2 & +K_{211} \\ 2 & 1 & -2 K_{212} & +2 K_{211} & -2 K_{222} \\ 2 & 2 & -2 K_{211} & +2 K_{212} & +2 K_{221}\end{array}$


Figure 1

Nonsecular change in length-of-day ( $\triangle \mathrm{LOD}$ ) in milliseconds vs. years $A D$, derived from subtracting a secular trend of $+1.6 \mathrm{~ms} \mathrm{cy}^{-1}$ due to a combination of tidal friction and (possibly) postglacial rebound from the total observed $\triangle \mathrm{LOD}$. Based on the work by $F$. R. Stephenson and L. V. Morrison (2).

\section{Figure 2}

Schematic of the density anomalies in the liquid outer core and the anomalies in the solid mantle, looking down from the north pole. The observed positive acceleration of the mantle over the last millenium (outer counterclockwise arrow) and the current westward drift of the core (inner clockwise arrow) may be due to internal gravitational torques between the mantle and core. 


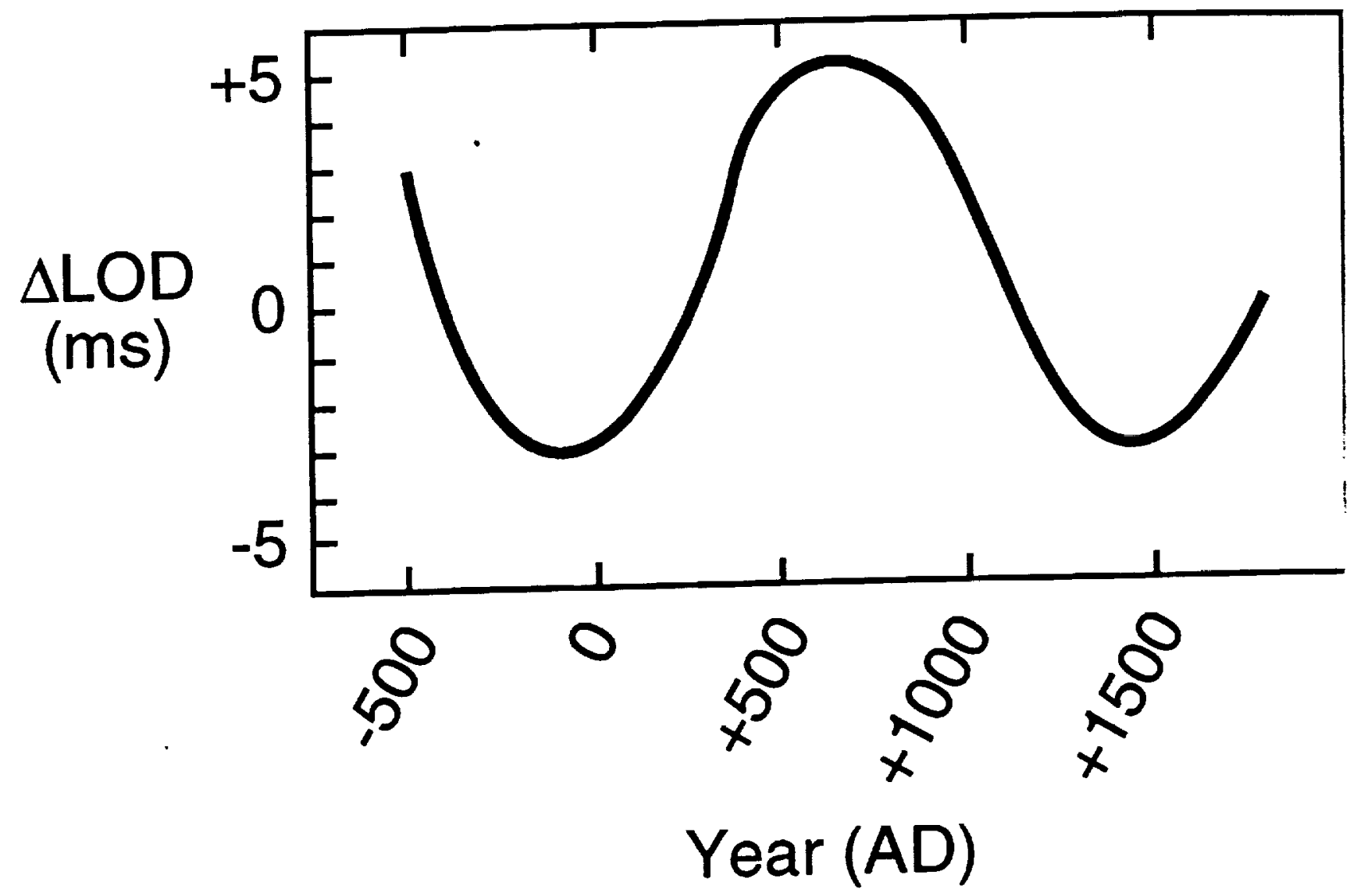

Figure 1 


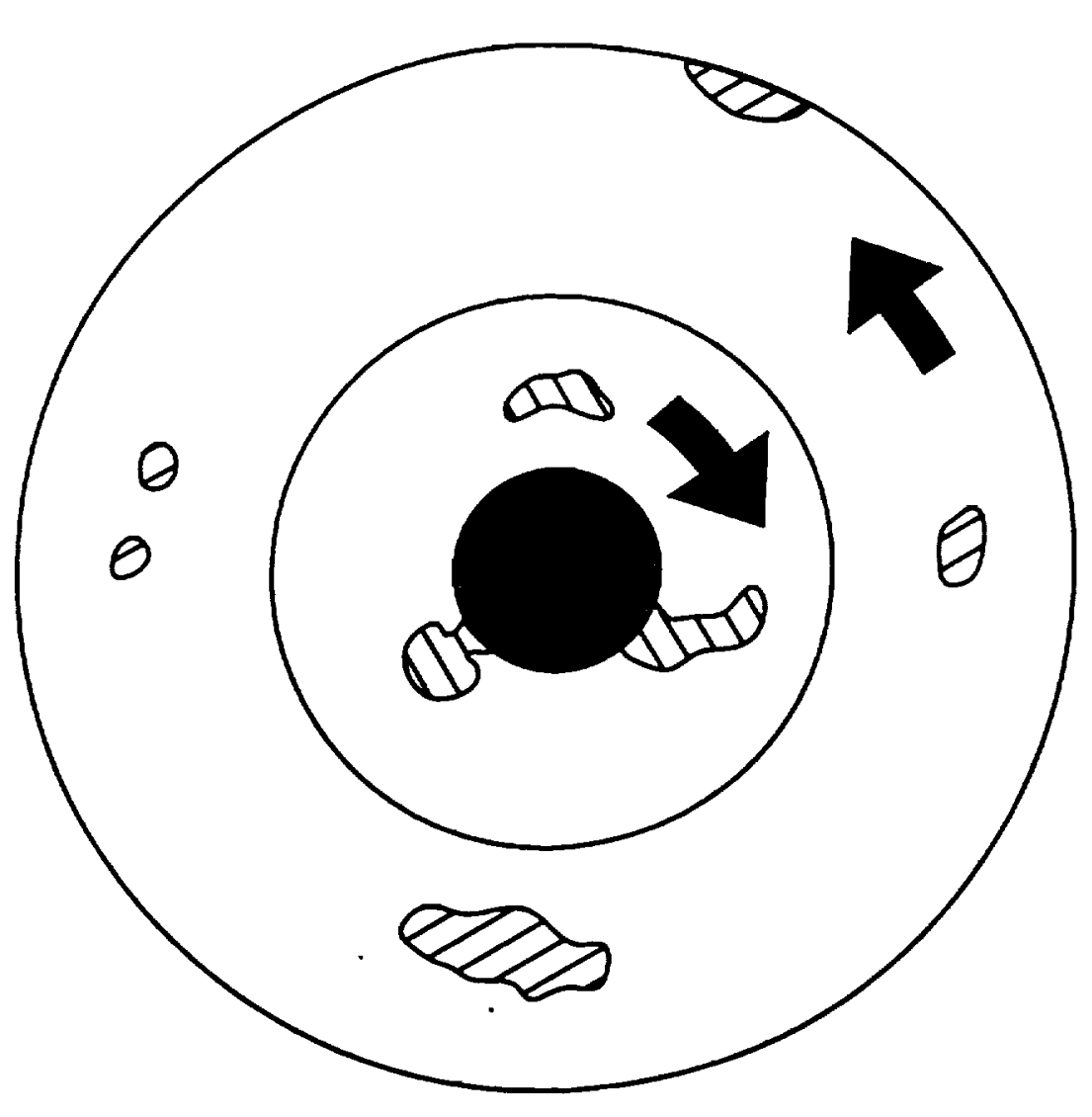

Figure 2 
Rubincam $\quad 3 / 27 / 01$

\section{Gravitational Core-Mantle Coupling and the Acceleration of the Earth}

\section{POPULAR SUMMARY}

The tides are slowing down the rotation of the Earth, overwhelming a smaller positive acceleration from postglacial rebound. According to historical records such as ancient and medieval observations of solar eclipses, the signal from these two secular effects appears to be significantly corrupted by a source (or sources) of an episodic or perhaps periodic nature operating on a 1000 year timescale. This nonsecular source causes accelerations which are the same order-of-magnitude as the tides, $\sim 6 \times 10^{-22} \mathrm{~s}^{-2}$. Coupling between the liquid outer core of the Earth and the mantle has long been a suspected reason for changes in the length-of-day. Research heretofore has been aimed at viscous, electromagnetic, and topographic coupling at the core-mantle boundary, mostly on the decadal timescale, and at gravitational coupling not related to the problem treated here.

The present investigation focusses on the gravitational coupling between the density anomalies in the convecting liquid outer core and those in the mantle and crust as a possible cause for the observed nonsecular acceleration on the millenial timescale. (Hereafter I use "mantle" as a shorthand for "mantle and crust.") The basic idea is as follows. There are density inhomogeneities caused by blobs circulating in the outer core like the blobs in a lava lamp; thus the outer core's gravitational field is not featureless. Moreover, these blobs will form and dissipate somewhat randomly. Thus there will be a time variability to the field. These density inhomogeneities will gravitationally attract the density anomalies in the mantle.

The outer core and mantle may not be in a state of gravitational equilibrium if the blobs form and dissipate faster than either the outer core or mantle can react to achieve a state of zero torque. In other words, the mantle and outer core may always be in a state of 
disequilibrium due to the stochastic nature of "core weather." The outer core and mantle will try to align with each other to make the torque between them zero; but before this can happen, the gravitational field of the outer core may change, so that the core and mantle never catch up with each other. Hence it may be that the outer core is forever torquing the mantle, changing its rotation state and perhaps producing the observed nonsecular acceleration and changing the length-of-day. By action-reaction the outer core will also change its rotation state.

A quantitative calculation indicates that the gravitational torque on the mantle from the liquid core may be sufficient to explain the accelerations and decelerations in the Earth's rotation on the 1000 year timescale. The corresponding torque by the mantle on the core may also explain the westward drift of the magnetic field of $0.2^{\circ}$ per year. Gravitational core-mantle coupling would stochastically affect the rate of change of the Earth's tilt by just a few per cent. Its contribution to polar wander would only be about $0.5 \%$ the presently observed rate of 10 centimeters per year. 